\title{
Presença do ossículo meniscal na articulação femorotibial de gatos-domésticos (Felis catus, Linnaeus, 1758)
}

\author{
Wiliam da Silva Prieto \\ Ana Carolina Fornari Borges de Carvalho \\ Luana Célia Stunitz da Silva * \\ Universidade Federal do Paraná, Setor Palotina \\ Rua Pioneiro, 2153, Jardim Dallas, CEP 85950-000, Palotina - PR, Brasil \\ * Autor para correspondência \\ luanastunitz@ufpr.br
}

Submetido em 03/02/2020

Aceito para publicação em 20/04/2020

\section{Resumo}

A articulação femorotibial é uma articulação complexa do tipo sinovial, que, além da relação com a patela, em algumas espécies de animais pode apresentar uma estrutura radiopaca visualizada ao exame radiográfico de formato oval entre os ossos do fêmur e tíbia, em projeção mediolateral, denominado ossículo meniscal. Embora comumente relatado em diferentes espécies, inclusive no homem, pouco se sabe sobre a sua função e frequência, sendo bem constatada sua existência em espécimes de grandes felídeos. O presente estudo teve como objetivo realizar a análise radiográfica da articulação femorotibiopatelar de 40 gatos-domésticos (Felis catus, Linnaeus, 1758) em projeções mediolateral e craniocaudal no intuito de avaliar a presença ou ausência do ossículo meniscal. Nas radiografias realizadas, o ossículo esteve presente em $15 \%(n=6)$, observando-se sua maior frequência em machos e unilateralmente, sendo o joelho esquerdo o de maior incidência da estrutura. Por ser um achado comum e relatado em diversas espécies selvagens de felídeos, a presença do ossículo meniscal deve ser levada em consideração e inserida como diagnóstico diferencial quando da realização de radiografias de gatos-domésticos e da visualização de opacidade intra-articular em projeções radiográficas mediolaterais da articulação femorotibiopatelar.

Palavras-chave: Artrologia; Felinos; Joelho; Lúnula

\section{Abstract}

Presence of meniscal ossicle in femorotibial joint of domestic cats (Felis catus, Linnaeus, 1758). The femorotibial articulation is a complex joint, that, on top of being related to the patella, may, in some animal species, present an oval-shaped radiopaque structure on radiographic examination, located between the femoral and tibial bones, in a mediolateral projection, called the meniscal ossicle. Although commonly related in different species, including humans, little is known about their function and frequency, and their occurrence in large felids is well reported. The present study aims to perform a radiographic analysis of the femorotibial articulation of 40 domestic cats (Felis catus, Linnaeus, 1758) in mediolateral and craniocaudal projections, and to evaluate the presence or absence of the meniscal bone and its frequency. From the radiographs taken, $15 \%(\mathrm{n}=6)$ of ossicle frequency were obtained, with higher frequency observed in males and unilaterally, the left knee being 
the one with the highest incidence of the structure. Because it is a common find reported in several wild felids species, the presence of the meniscal ossicle should be considered and included as a differential diagnosis when performing radiographs of domestic cats and visualizing intra-articular opacity in mediolateral radiographic projections of the femorotibial articulation.

Key words: Arthrology; Feline; Lunulae; Stifle

\section{Introdução}

A articulação femorotibiopatelar, ou joelho, é a articulação mais complexa do corpo, sendo considerada uma articulação do tipo sinovial, permitindo a realização de movimentos de extensão e flexão do membro pélvico (MONTEIRO et al., 2012). Composta pelas articulações femorotibial, femoropatelar, tibiofibular proximal e seus respectivos ligamentos, nos cães e gatos também estão incluídas as articulações entre o osso fêmur e o par de ossos sesamoides localizados na origem do músculo gastrocnêmio e entre a tíbia e o único osso sesamoide do músculo poplíteo. Ressalta-se que na espécie felina todas essas articulações compartilham uma cavidade sinovial comum (DYCE et al., 2010). Apesar da complexidade o joelho funciona como uma dobradiça, cuja movimentação livre é restrita à flexão e à extensão, tendo sua estabilidade mantida principalmente pelos ligamentos cruzados (DYCE et al., 2010; KONIG; LIEBICH, 2016). Outra importante estrutura que compõe a articulação é o par de meniscos, estruturas fibrocartilaginosas e semilunares localizadas entre os côndilos do fêmur e os da tíbia, unidos cranial e caudalmente em gatos por ligamentos, conferindo à articulação apoio e estabilidade, uma vez que compensam a incongruência das superfícies articulares (MONTEIRO et al., 2012; KONIG; LIEBICH, 2016).

Em alguns animais, um osso sesamoide adicional chamado de lúnula ou ossículo meniscal, descrito desde 1921, também pode estar presente (PEARSON; DEVIN, 1921; WALKER et al., 2002; FREIRE et al., 2010). Esse pequeno osso sesamoide adicional foi relatado em uma variedade de espécies animais incluindo répteis, aves, anfíbios e mamíferos, tais como algumas espécies de felídeos, primatas não humanos e poucos humanos (PEARSON; DEVIN, 1921). Anatomicamente, os ossículos meniscais são vascularizados a partir da periferia do menisco e suas superfícies proximal e distal são cobertas por cartilagem hialina. Não apresentam medula óssea e são radiograficamente evidentes desde um ano de idade (FREIRE et al., 2010). Teorias para designar a etiologia da mineralização intrameniscal e ossificação incluem condições filogenéticas, variações anatômicas, sequelas de traumas ou processos degenerativos e centros de ossificação primários normais (WALKER et al., 2002; LINO et al., 2018). Uma possível explicação de sua função seria a de preservar a articulação frente a desgastes e promover melhor movimentação do menisco em movimentos de extensão e extensão máxima da articulação (WALKER et al., 2002).

Radiograficamente, os ossículos meniscais podem aparecer com formato oval radiopaco no compartimento cranial, situando-se na face cranial entre o fêmur e a tíbia em uma projeção mediolateral (CERVENY; PÁRAL, 1995). Em projeção craniocaudal, a mineralização é mais difícil de ser observada (RAHAL et al., 2013). Baseado nisso, o presente estudo objetivou avaliar, mediante exame radiográfico simples da articulação femorotibial, a presença do ossículo meniscal em um grupo de gatos-domésticos.

\section{Material e Métodos}

No presente estudo foram utilizados 40 gatosdomésticos (Felis catus), sendo 22 indivíduos machos $(55 \%)$ e 18 fêmeas (45\%), em sua grande maioria sem raça definida (SRD), tendo apenas um indivíduo da raça Persa. Todos possuíam idade inferior a seis anos, e, destes, três animais eram menores de um ano. Os animais eram provenientes da rotina clínica do Hospital Veterinário da Universidade Federal do Paraná - Setor Palotina e de colaboradores deste. Para a realização das projeções mediolaterais de todas as 80 unidades articulares separadamente e das 40 projeções craniocaudais, foi utilizada a sala de radiografia do Setor de Diagnóstico por Imagem do Hospital Veterinário da 
Universidade Federal do Paraná, situado em Palotina - PR, de acordo com os princípios éticos de bem-estar animal e aprovadas pela Comissão de Ética no uso de animais da referida instituição, Protocolo no 16/2018.

Foi utilizado um aparelho radiográfico da marca Diafix $500 \mathrm{~mA}$, modelo $125 \mathrm{kV}$, com digitalizador CR30-X e com Regulador Fuji para as imagens. Todos os animais foram submetidos à avaliação radiográfica simples, sem sedação, de ambas as articulações femorotibiais. Em todos os exames os animais foram posicionados em decúbito lateral, para realização das projeções mediolaterais, e em decúbito dorsal com extensão dos membros pélvicos, para a aquisição das projeções craniocaudais de ambos os joelhos. Nessa última optou-se pela aquisição das imagens em uma única projeção com ambas as articulações expostas. Todas as imagens geradas foram coletadas e analisadas mediante o Software Radiant ${ }^{\circledR}$ por um mesmo avaliador.

À avaliação do exame radiográfico, estabeleceu-se como parâmetro avaliativo a presença ou ausência da estrutura óssea compatível com o ossículo meniscal, considerando positivo quando presente foco de radiopacidade mineral em topografia radiográfica de meniscos de um ou ambos os joelhos, e negativo quando não observado tal achado (Figura 1). Salienta-se que foi descartado do estudo quaisquer animais e unidades articulares que apresentassem sinais de doença articular ativa.

\section{Resultados}

Foram encontradas evidências da presença do ossículo meniscal em seis (15\%) dos 40 pacientes avaliados (Figura 2), sendo que destes a maioria $(n=4)$ demonstrou a presença unilateral da estrutura $(66,7 \%)$, tendo apenas dois pacientes com achados em ambos os joelhos $(33,3 \%)$, representados na população geral do grupo estudado como $10 \%$ e $5 \%$ respectivamente. A visualização dos ossículos foi possível apenas nas radiografias em projeção mediolateral. Ocorreu uma maior incidência sobre os machos, $(5 / 6,83,3 \%)$ e entre os indivíduos houve a presença em um animal com menos de um ano de idade. A frequência de aparecimento do achado entre os joelhos foi maior do lado esquerdo, sendo que houve a presença do ossículo em quatro unidades articulares esquerdas e em três unidades articulares direitas. Nos demais indivíduos avaliados, os ossículos foram classificados como ausente neste estudo.

FIGURA 1: Projeção radiográfica ortogonal simples da articulação femorotibiopatelar de gatos-domésticos com ausência do ossículo meniscal. A) Projeção mediolateral. B) Projeção craniocaudal.

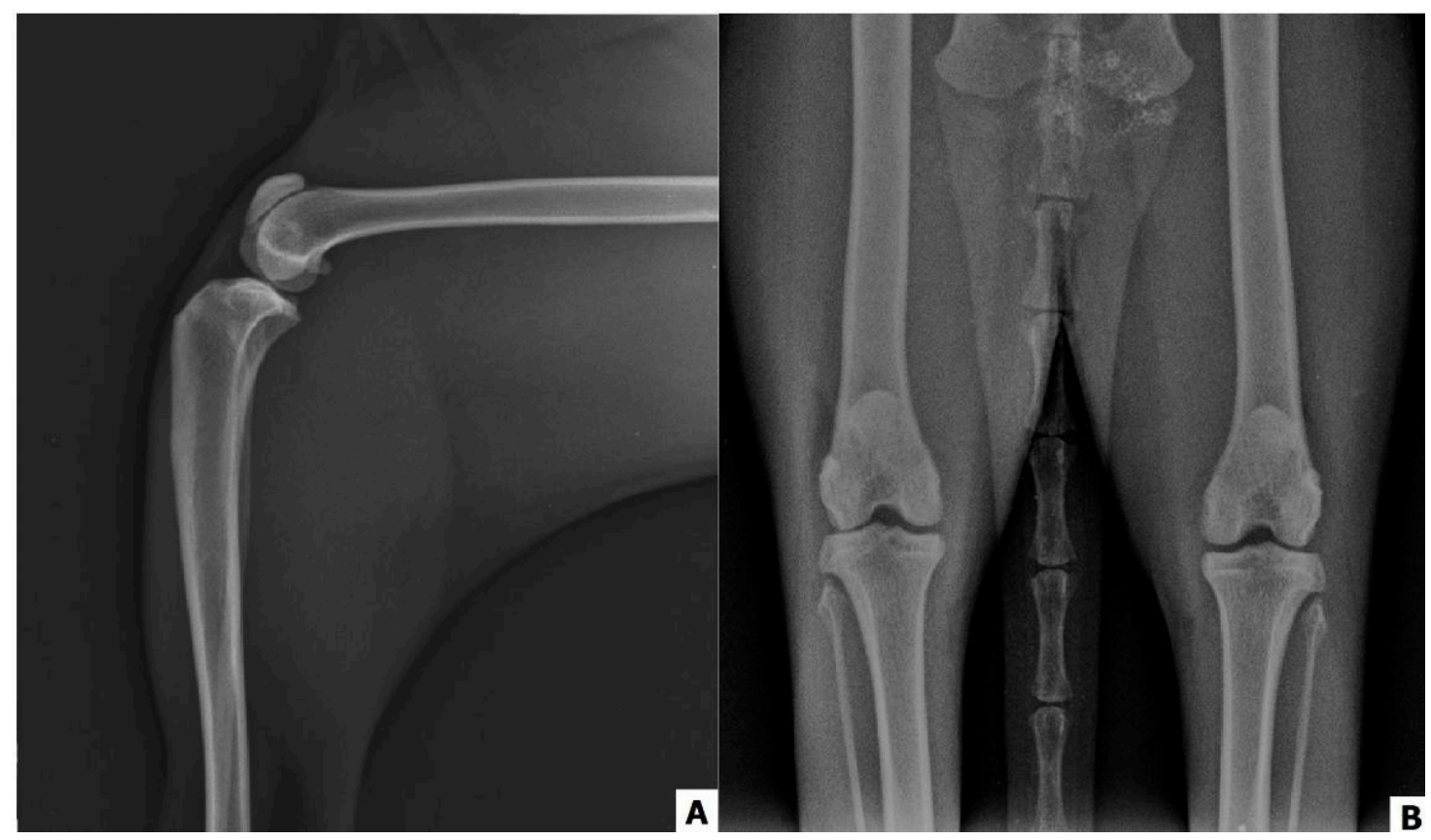


FIGURA 2: Projeção radiográfica mediolateral simples de articulação femorotibiopatelar em gatos-domésticos (Felis catus), evidenciandose a presença do foco de mineralização (seta vermelha). A: macho, 1 ano e 1 mês. B: macho, 1 ano e 5 meses.

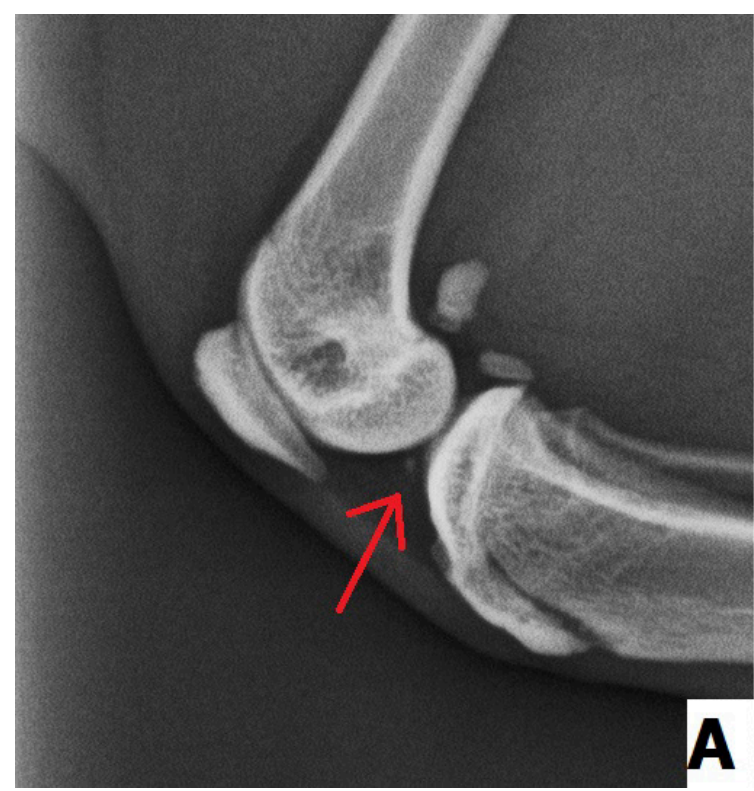

\section{Discussão}

Embora seja um achado reportado há tempos em diferentes espécies, inclusive no homem, pouco se sabe sobre a causa e finalidade da presença do ossículo meniscal (WALKER et al., 2002; FREIRE et al., 2010; LINO et al., 2018), sendo que, em busca de medidas explicativas, teorizam-se diversas possibilidades sobre o seu surgimento (LINO et al., 2018). Uma das possibilidades levantadas é de que o ossículo seja um resquício evolutivo e algumas das possíveis funções dessa estrutura óssea seriam a de facilitar o movimento cranial do menisco quando se aumenta a carga sobre a articulação durante a extensão máxima do joelho, bem como para facilitar a prevenção do desgaste das superfícies articulares de fêmur e tíbia quando de uma mudança no formato dos meniscos durante o movimento de extensão do membro pélvico (WALKER et al., 2002; LINO et al., 2018).

Diversos autores demonstram a comum presença desse achado em espécies de grandes felídeos selvagens, tais como em leões africanos (Panthera leo) (WALKER et al., 2002), tigres-de-bengala (Panthera tigris tigris) (GANEY et al., 1994), leopardos (Panthera pardus) (WALKER et al., 2002), onças-pardas (Puma concolor)

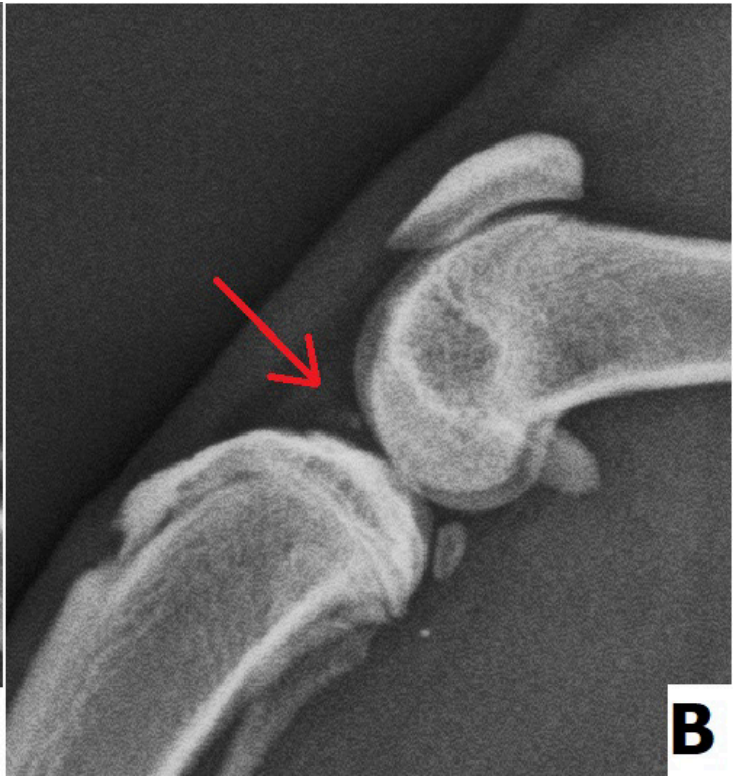

(CERVENY; PÁRAL, 1995), onças-pintadas (Panthera onca) (WALKER et al., 2002) e gatos-do-mato (Leopardus tigrinus) (RAHAL et al., 2013). Uma vez que os grandes felídeos são espécies que mantêm seus hábitos selvagens e dependem da flexão e extensão do joelho para correr e saltar, tais ossículos meniscais podem estar presentes frente a essa necessidade, ao contrário do que é observado em gatos-domésticos, então, devido a isso, muitos destes podem não ter mais a presença da estrutura (WALKER et al., 2002), tal como evidenciado no estudo em questão. Em guepardos (Acinonyx jubatus) esse achado também é comumente encontrado e alguns autores correlacionam que possa estar associado à angulação da articulação, que nesses animais é de $55^{\circ}$ (GROENEWALD et al., 2000). Em estudo morfológico da articulação de um tigre evidenciou-se, além da presença do ossículo, a existência de um sulco em côndilo femoral medial que se articularia com o ossículo meniscal medial (GANEY et al., 1994; FREIRE et al., 2010).

Outra possibilidade reportada para o desenvolvimento do ossículo meniscal seria o envolvimento da estrutura a um processo degenerativo da articulação, como em processos de osteoartrites (LINO et al., 2018), sendo que na presença destes poderia haver a formação 
de osteófitos (THRALL, 2014), anormalidades estas que não foram visualizadas em nenhum dos gatosdomésticos analisados neste estudo. Em contrapartida à teoria mencionada, há relatos da presença dos ossículos meniscais em animais jovens, e no estudo em questão um dos indivíduos positivos para o achado apresentava idade inferior a um ano, idade essa em que as chances da presença de um processo degenerativo são ínfimas (WALKER et al., 2002; LINO et al., 2018). Além disso, em estudo conduzido por Freire et al. (2010), o escore de dor, quando se comparava animais sem alterações radiográficas do joelho com os que apresentavam o achado de mineralização meniscal, não demonstrou diferença significativa. Em outros estudos com felídeos silvestres observou-se a ocorrência do ossículo sem achados de doença articular associados ao exame radiográfico (RAHAL et al., 2013), assim como observado no estudo ora conduzido, em que as unidades articulares avaliadas não apresentavam sinais radiográficos de doença articular concomitante à presença do ossículo meniscal. Entretanto, havendo a presença desse achado em animais de meia-idade, não se pode descartar a real presença de algum processo inflamatório ou degenerativo, por isso salienta-se que diagnósticos diferenciais para opacificações por calcificações intra-articulares devem ser feitos, incluindo na lista de diferenciais a osteocondrite dissecante, avulsão óssea, mineralização distrófica de tecido mole, osteocondroma sinovial, assim como os ossículos meniscais (WALKER et al., 2002). Em estudo realizado por Freire et al. (2010), observou-se que os animais com mineralização meniscal apresentavam mais danos à cartilagem de côndilo femoral medial e platô tibial, não se estabelecendo se a mineralização apresentava correlação como causa ou consequência dessa condição, porém sugere-se que gatos que apresentem alteração na marcha e no padrão de deambulação ou ainda alterações angulares do membro podem promover adução excessiva da articulação e culminar com o desenvolvimento da mineralização e esse ser um indício de uma artropatia incipiente.

Outra explicação seria a ocorrência de focos de ossificação heterotópica em resposta a sequelas póstraumáticas, tais como processos inflamatórios ou traumas crônicos que induzem à atividade osteogênica e proliferação celular culminando na calcificação, sendo que em resposta a esses processos o tecido fibrocartilaginoso já formado pode retornar a seu estado mesenquimal, sucedendo à osteogênese, ou induzir a metaplasia tecidual (LINO et al., 2018). Tais processos são favorecidos em condições de vascularização abundante, assim como os cornos meniscais, que demonstram maior vascularização e inervação se comparados ao centro do menisco, corroborando a condição de que os ossículos meniscais são achados localizados com maior frequência em corno cranial do menisco medial, achado este reportado em guepardos e em algumas espécies de tigres (WALKER et al., 2002; LINO et al., 2018).

A última teoria, essa por sua vez mais relatada em humanos, inclui correlação da existência dessas formações a lesões traumáticas antigas, sugerindo que as estruturas presentes na articulação sejam fragmentos ósseos de origem tibial (LINO et al., 2018).

A frequência desse achado em gatos-domésticos ainda é desconhecida, carecendo de uma amostragem maior para elucidação desse parâmetro, porém sabe-se que a presença do ossículo meniscal durante a avaliação ortogonal simples da articulação femorotibial é uma característica comum detectada, pois em um estudo com 100 gatos-domésticos obteve-se uma frequência positiva de 37\% (FREIRE et al., 2010), embora no presente estudo a frequência tenha sido menor (15\%), frente também à população estudada também menor. Não há fatores que expliquem a maior prevalência sobre os machos ou sobre qual antímero (esquerdo ou direito) há um maior acometimento, como ora observado, porém alguns trabalhos relatam haver maior incidência de forma bilateral (FREIRE et al., 2010; RAHAL et al., 2013). Não podendo se descartar a possibilidade de que, nos gatos-domésticos em que havia unilateralidade do ossículo, o ossículo do joelho contralateral ainda não fosse visível radiograficamente, uma vez que em um estudo histológico de meniscos em cadáveres de gatos-domésticos relatou-se prevalência de áreas de calcificação em todos os animais estudados, sendo que uma possível causa da visualização unilateral da estrutura ora observada pode estar correlacionada com a idade do grupo de indivíduos avaliados, todos 
com menos de seis anos (FREIRE et al., 2010). Salienta-se que os animais positivos para a presença da mineralização intrameniscal devido as suas idades, quando do momento das análises radiográficas, não apresentavam quaisquer alterações compatíveis com doença articular degenerativa, não estando ilesos dessa patologia em idades avançadas. Ademais quando presente, o exame radiográfico possui alta capacidade na detecção do ossículo que, aliado à sua disponibilidade, pode ser considerado um bom exame auxiliar para o diagnóstico do ossículo meniscal mediante projeção mediolateral, enquanto que a projeção craniocaudal demonstra pouca sensibilidade e maior dificuldade (FREIRE et al., 2010; RAHAL et al., 2013).

\section{Referências}

CERVENY, C.; PÁRAL, V. Sesamoid bones of the knee joint of the Puma concolor. Acta Veterinaria BRNO, Brno, v. 64, p. 79-82, 1995.

DYCE, K. M.; SACK, W. O.; WENSING, C. J. G. Tratado de anatomia veterinária. 4. ed. Rio de Janeiro: Guanabara Koogan, 2010. $856 \mathrm{p}$.

FREIRE, M.; BROWN, J.; ROBERTSON, I. D.; PEASE, A. P.; HASH, J.; HUNTER, S.; SIMPSON, W.; SUMRELL, A. T.; LASCELLES, D. X. Meniscal mineralization in domestic cats. Veterinary Surgery, Germantown, v. 39, p. 545-552, 2010.
GANEY, T. M.; OGDEN, J. A.; ABOU-MADI, N.; COLVILE, B.; ZDYZIASRSKI, J. M.; OLSEN, J. H. Meniscal ossification II. The normal pattern in the tiger knee. Skeletal Radiology, Heidelberg, v. 23, p. 173-179, 1994.

GROENEWALD, H. B.; WAGNER, W. M.; KIRBERGER, R. M. A radiological study of the sesamoid bones and os meniscus of the cheetah (Acinonyx jubatus). Veterinary and Comparative Orthopaedics and Traumatology, Stuttgart, v. 13, n. 4, p. 172177,2000

KONIG, H. E.; LIEBICH, H. Anatomia dos animais domésticos: texto e atlas colorido. 6. ed. Porto Alegre: Artmed, 2016. 824 p.

LINO, H. A.; FARIA, B. L.; CUNDARI, A. M. M. V. Osso meniscal: uma revisão da literatura. Revista Médica de Minas Gerais, Belo Horizonte, v. 28, p. 1-7, 2018.

MONTEIRO, B. S.; ZANELLA, A. R.; SANTOS, B. S.; FARIA, R. D.; BATISTA, A. S. Lesões meniscais em pequenos animais. Arquivos de Ciências Veterinárias e Zoologia da UNIPAR, Umuarama, v. 15, n. 2, p. 143-148, 2012.

PEARSON, K.; DAVIN, A. G. On the sesamoids of the knee joint, part II. Evolution of the sesamoids. Biometrika, Oxford, v. 13, n. 2-3, p. 133-175, 1921.

RAHAL, S. C.; FILLIPI, M. G.; MAMPRIM, M. J.; OLIVEIRA, H. S.; TEIXEIRA, C. R.; TEIXEIRA, R. H. F.; MONTEIRO, F. O. B. Meniscal mineralisation in the little spotted cats. BMC Veterinary Research, London, v. 9, p. 2-6, 2013.

THRALL, D. E. Diagnóstico de Radiologia Veterinária. 6. ed. Rio de Janeiro: Elsevier, 2014. 864 p.

WALKER, M.; PHALAN, D.; JENSEN, J.; JOHNSON, J. Meniscal ossicles in large non-domestic cat. Veterinary Radiology \& Ultrasound, Cleveland, v. 43, n. 3, p. 249-254, 2002. 